\title{
Inhibiting prepared and ongoing responses: Is there more than one kind of stopping?
}

\author{
SHARON MOREIN-ZAMIR, PAUL NAGELKERKE, ROMEO CHUA, \\ IAN FRANKS, and ALAN KINGSTONE \\ University of British Columbia, Vancouver, British Columbia, Canada
}

\begin{abstract}
Inhibiting movements has been investigated widely using the countermanding (stop signal) paradigm. Although it has been assumed that response inhibition, as measured by the countermanding task, generalizes to all forms of stopping, this has never been tested. In the present study, stopping performance in the countermanding paradigm was compared with stopping performance in a new paradigm in which a continuous-tracking task was used. Although stimulus presentations were matched across paradigms, the two tasks differed in the type of stopping required. In the countermanding paradigm, response inhibition latency was measured prior to response execution-this is, it was inferred from the successful withholding of a $g o$ response. In the new paradigm, response inhibition was carried out after response execution-that is, it was measured as the time to begin stopping a continuous tracking response. Results indicated that stopping latencies between the two paradigms were highly correlated, providing strong evidence that stopping an unexecuted response engages the same mechanisms as stopping an ongoing response.
\end{abstract}

By inhibiting a response, we can deliberately stop ourselves from enacting an undesired behavior, such as voicing an inappropriate remark. There is much appeal to studying direct measures of response inhibition, since this can lead to insights into how we control our actions, how this control changes with age, and how it is affected by deficiencies in response inhibition (Kok, 1999; Kramer, Humphrey, Larish, Logan, \& Strayer, 1994; Logan, 1994; Oosterlaan, Logan, \& Sergeant, 1998). In the laboratory, stopping has been studied extensively with the countermanding procedure (Lappin \& Eriksen, 1966; Logan, 1994; Logan \& Cowan, 1984). Participants receive a response signal on every trial, and occasionally, a stop signal follows the go signal, indicating that the response should be withheld. By varying the delay between the go signal and the stop signal, the probability of responding is manipulated: A short delay results in many successful stops; a long delay results in few stops (e.g., Slater-Hammel, 1960).

Performance in this task is well described by a horse race model between go and stop processes (Logan \& Cowan, 1984; Logan, Cowan, \& Davis, 1984; Osman, Kornblum, \& Meyer, 1990; but see also McGarry, Inglis, $\&$ Franks, 2000). If the go process wins the race, there is a response, but if the stop process wins, no response occurs. By assuming that the two processes have independent finishing times, the race model allows an estimate

This research was supported by grants to A.K. from the Human Frontier Science Program, NSERC, and the Michael Smith Foundation of Health Research, and to I.F. from NSERC. Correspondence should be addressed to S. Morein-Zamir, Psychology Department, University of British Columbia, 2136 West Mall, Vancouver, BC, Canada V6T 1 Z4 (e-mail: smorein@interchange.ubc.ca). of stop signal reaction time (SSRT) to be derived even though no overt action of stopping was observed (Band, van der Molen, \& Logan, 2003; Logan, 1994). Interestingly, SSRTs typically converge around 200-300 msec (De Jong, Coles, \& Logan, 1995; Logan, 1994; Logan \& Cowan, 1984). This convergence has led to the conclusion that countermanding SSRT measures a generic stopping process (Band \& van Boxtel, 1999; Logan, 1994; van Boxtel, van der Molen, Jennings, \& Brunia, 2001). ${ }^{1}$

This conclusion is quite bold, considering that to date, the evidence has been derived almost exclusively with a countermanding task, which by definition can only address stopping a response before it is executed. Thus, a more conservative conclusion based on the extant data would be that a generic stopping process is engaged when one has to inhibit a response before it is carried out. Whether the stopping process that is being measured for an unexecuted response is generic to other forms of stopping is an open question. This issue is important for the development of theories of response inhibition that are grounded in the countermanding procedure, as well as with regard to the generalizability of the countermanding procedure (Barkley, 1997; Logan, 1994; Logan \& Cowan, 1984; Nigg, 2000). Indeed, recent theories of inhibition distinguish between several subsets of inhibition, suggesting that effortful inhibition of motor responses is not a unitary construct (Nigg, 2000). Hence, it is possible that stopping at various stages of action planning and execution or stopping different actions altogether would engage different mechanisms and be subject to different constraints.

To measure whether stopping is a generic process, one must measure different forms of response inhibition. In 
the present study, the negation of a response before it was executed (i.e., countermanding) was measured, as well as the negation of a response that was already being executed. The latter form of stopping, which by definition cannot be measured using the classic countermanding task, occurs routinely in everyday life. Whether it is stopping in midstride at the edge of a crosswalk when the light changes to red or stopping in midsentence when the lecturer looks one's way, our daily lives are rife with ongoing responses that must be halted (see also Ladefoged, Silverstein, \& Papcun, 1973).

Measuring the termination of an action after it is already underway can be done with a simple pursuit task (Morein-Zamir \& Meiran, 2003). After a continuoustracking response has been initiated, a stop signal is presented, indicating to participants that they must stop their tracking response as quickly as possible. Tracking SSRTs are obtained by measuring the time from stop signal onset to the initial stopping or deceleration of the ongoing response (Henry \& Harrison, 1961; Morein-Zamir $\&$ Meiran, 2003). To compare the countermanding and the pursuit tasks, we matched procedures so that the two tasks differed only in the type of stopping that was required. Thus, the perceptual characteristics of both tasks were highly comparable, as were the attentional requirements. We questioned whether the two forms of stopping shared a common response inhibition mechanism by examining whether stopping performance was correlated across the two tasks. In addition, we examined whether there would be any consistent differences between the stopping measures. In particular, we explored whether SSRTs would be of the same magnitude across tasks and whether stop signal delay would influence SSRTs in the same manner.

In sum, the countermanding task is limited to measuring a particular type of stopping - the inhibition of an unexecuted response. The assumption, however, heretofore untested, is that this countermanding measurement reflects a process of inhibition that is common to all forms of stopping, including the inhibition of an executed response. The present study put this crucial issue to the test.

\section{METHOD}

\section{Participants}

Ten undergraduates ( 8 female) participated; mean age was 19.7 years $(S D=1.33)$. All had normal or corrected-to-normal vision, and all but one were right-handed.

\footnotetext{
Apparatus and Stimuli

In both tasks, the display was a green response circle (radius, $0.3^{\circ}$ ) and a blue target ring (radius, $0.3^{\circ}-0.405^{\circ}$ ) moving around in an $8^{\circ}$ radius circular path. A stimulus flash consisted of a white square $\left(0.81^{\circ} \times 0.81^{\circ}\right)$ appearing directly over the target. The stimuli were presented on a 14 -in. VGA monitor $(640 \times 480$ pixel resolution, $60-\mathrm{Hz}$ refresh). Responses were executed using a telegraph key instrumented with two strain gauges. Analogue signals from the strain gauges were amplified (Northwood Instruments, Model IA-102-500) and were sampled at $1000 \mathrm{~Hz}$ by an A/D converter (Techmar Labmaster).
}

\section{Procedure}

The participants completed two sessions of 480 trials on consecutive days (task order counterbalanced). Stop and go trials were presented randomly, with self-terminating breaks every 48 trials.

In the countermanding task, each trial started with the stimuli stationary at the 3 o'clock position of an imaginary circle (see Figure 1). After a random delay of 200-600 msec, the stimuli began circling in unison at $0.5 \mathrm{~Hz}$. A $100-\mathrm{msec}$ flash occurred $2.5-3.5 \mathrm{sec}$ later (determined randomly). The participants were instructed to attend to the stimuli and to press the response key as quickly as possible when the flash appeared. The key was adjusted so that switch closure occurred with $1 \mathrm{~mm}$ of key travel with a 100-g mass. The stimuli continued to rotate until $5 \mathrm{sec}$ had elapsed from trial onset. These go trials composed $75 \%$ of the trials. On the remaining stop trials, the stimuli stopped at equal probabilities 30,110, 190, or $270 \mathrm{msec}$ after flash onset and remained stationary until the trial ended. In each block of 48 trials, there were 3 stop trials in each delay. The participants were informed that on some trials the circles would stop (the stop signal) and that they were to stop and not press the key. They were also instructed at the beginning and midway into the session not to delay responses to the flash in order to improve their chances of stopping (see Logan, 1994, p. 223, for instruction details).

The sequence of events in the tracking task was effectively identical, with $25 \%$ stop trials and the same delays between the flash and the stop signal as before. At the beginning of the trial, the two stimuli appeared stationary, and after the randomly determined period, the target marker began to move. Now however, the participants controlled the speed of the response circle (the target was still controlled by the program). The response circle speed increased when the participants increased their pressure on the key. A fixed pressure of $400 \mathrm{~g}$ yielded a speed of $0.5 \mathrm{~Hz}$, minimum pressure was $100 \mathrm{~g}(0.125 \mathrm{~Hz})$, and maximum pressure was $2,000 \mathrm{~g}(2.5 \mathrm{~Hz})$. The participants were instructed to move the response stimulus to overlap the target, but also to stop as quickly as possible if the target stopped. Stopping was stressed as releasing the pressure, without lifting the finger from the key to ensure maximal similarity between resident and remote effects (Hommel, Müsseler, Aschersleben, \& Prinz, 2001; see the discussion below). The flash occurred as in the countermanding task but was irrelevant to the task.

The main difference between the procedures was that in the countermanding task, the response and the target stimuli were always aligned, whereas in the pursuit task, the participants controlled the response stimulus alignment with the target. Typically, there was considerable overlap between the two stimuli as the participants tracked successfully. In addition, the flash in the countermanding task served as both a go signal and a temporal warning stimulus that the stop signal might occur soon. In the tracking task, the flash served only as a warning stimulus for the stop signal.

\section{RESULTS AND DISCUSSION}

\section{Computing the Dependent Measures}

The nature of the two tasks demanded that different data be collected. In the countermanding task, go-RTs to the flash were measured for each participant, as was probability of response in each delay (mean inhibition probability was .53). SSRT was estimated by first rank ordering go-RTs in trials in which no stop signal was presented, then determining the $n$th RT, where $n$ is the probability of responding in a given delay multiplied by the number of go-RTs. This produced an estimate of the time required to stop, relative to the onset of the flash. By subtracting the delay, SSRT was obtained for that particular delay (Logan, 1994). For example, if the prob- 


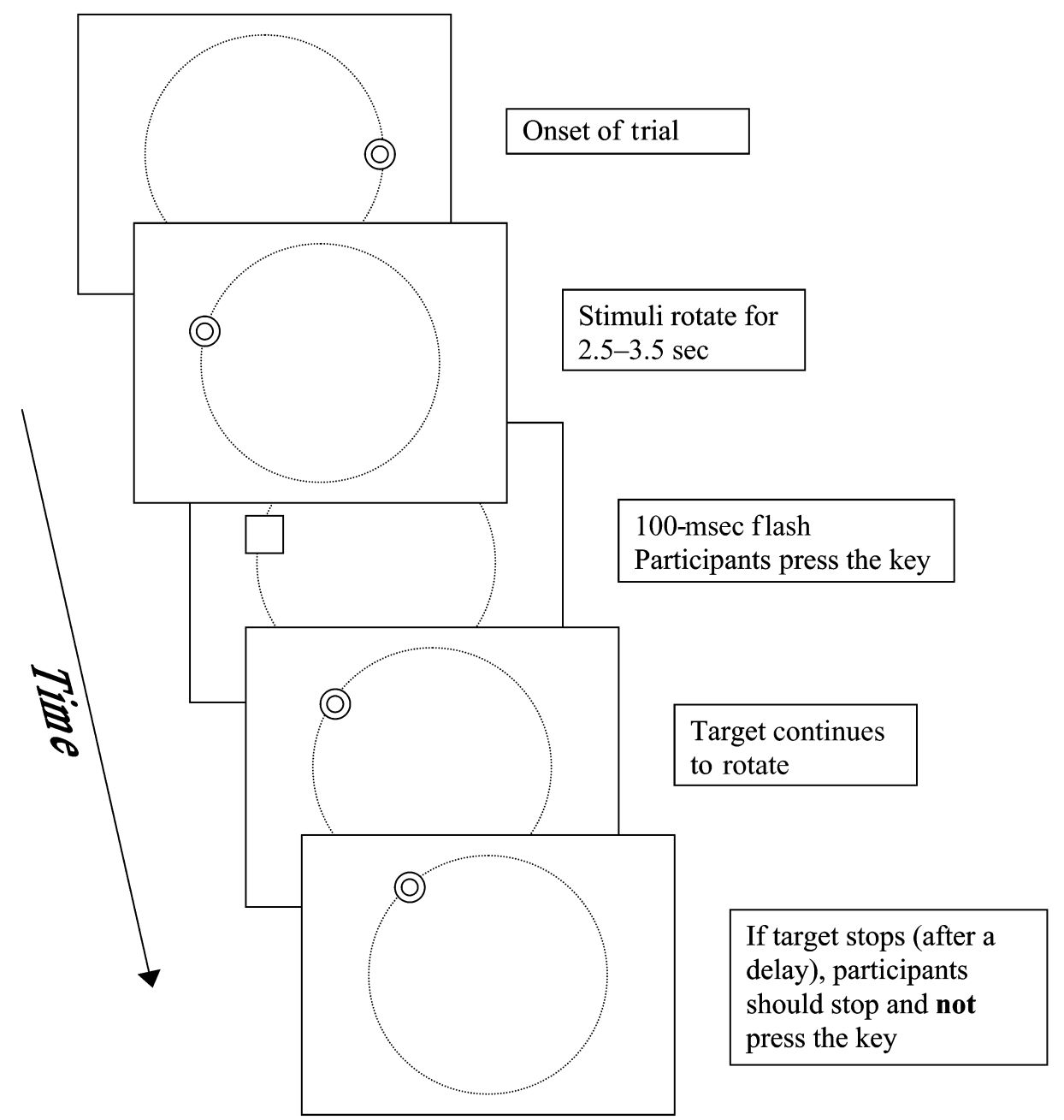

Figure 1. A schematic depiction of the sequence of events on a stop trial during the countermanding task. The stimulus and response rings rotate along an imaginary circle, illustrated by the dashed line. The participants are instructed to press the key in response to the flash. The stimuli continue to rotate until the end of the trial. On stop trials only, the events in the last frame occur, where the stimuli stop abruptly, indicating that the participants should withhold their keypress response. The tracking task is highly similar; however, the participants control the response stimulus speed by pressing the key. On stop trials in this task, the stimulus ring stops, signaling that the participants should stop pressing the key.

ability to respond at a delay of $30 \mathrm{msec}$ for Participant 1 was .15, the SSRT for that delay was calculated by subtracting the delay value from the 15 th percentile of the go-RT (e.g., subtracting $30 \mathrm{msec}$ from $300 \mathrm{msec}$, yielding $270 \mathrm{msec}$ ). Thus, SSRTs for individual delays were not calculated by averaging. $\mathrm{SSRT}_{1}$ was determined by averaging across the delays. An alternative method for estimating SSRT subtracts the mean of the inhibition function from the mean go-RT to yield $\mathrm{SSRT}_{2}$. The mean of the inhibition function was computed by multiplying each stop signal delay with the probability of responding at the $i$ th delay minus the probability of responding at the $i-1$ th delay (Logan, 1994). The two SSRTs were then averaged to attain the most reliable measure of countermanding SSRT (Logan \& Cowan, 1984). ${ }^{2}$
In the tracking task, a profile of the pressure on the response key was attained for each trial (see Figure 2). Tracking performance was measured by phase error (PE; i.e., the separation in degrees, along the diameter of the imaginary circle, between the target and the response stimuli). PE was sampled at $1000 \mathrm{~Hz}$ for $1 \mathrm{sec}$ before the flash and was averaged for that duration. Hence, the smaller the PE, the better the participants were at tracking the target. On stop trials, tracking SSRT measured the time from stop signal onset to the initiation of pressure offset, and final RT measured the time from stop signal onset to the termination of response pressure. The algorithm computing tracking SSRT used a threshold of $250 \mathrm{~g}$ to find the middle of the offset curve and then worked backward to find a slope value of less than $10 \%$ 


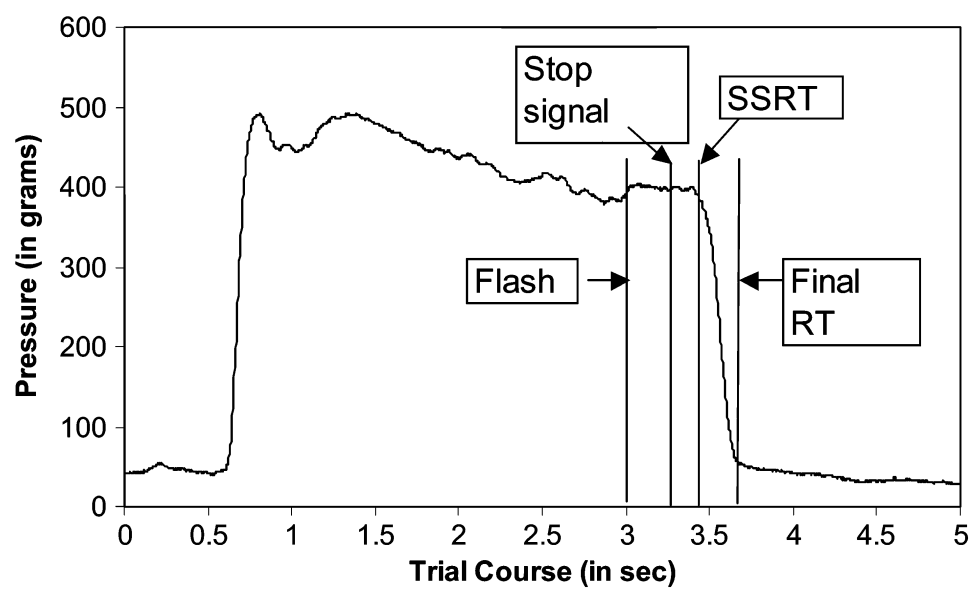

Figure 2. An example of the response pressure profile of a stop trial in the tracking task. At first, as the target was stationary and the participant rested his or her finger, the response pressure was below the minimum necessary for motion. After the target began to move, the participant pressed, yielding a steep elevation in pressure that was maintained as the target continued to rotate. Following the stop signal, the pressure decreased abruptly, falling to a resting level until the end of the trial. The vertical lines (from left to right) represent the flash onset, stop signal onset, time detected by the algorithm as stop signal reaction time (SSRT), and time detected as final RT.

peak slope. The same algorithm detected final RT by moving forward from the curve center to find a slope value of less than $10 \%$ of peak slope. ${ }^{3}$

\section{Is There a Shared Response Inhibition}

\section{Mechanism? Do the SSRT Measures Converge?}

Correlations were computed between all dependent measures (see Table 1). The crucial result is the significant positive correlation of .84 between countermanding SSRT and tracking SSRT (for all significant correlations, $p<.05) .{ }^{4}$ A person who is fast and efficient at stopping an action before response initiation is fast and efficient at stopping an action already being executed. The high correlation provides compelling evidence that performance in the two tasks was mediated by the same inhibitory mechanism. This result supports the conclusion that the response inhibition measured here applies across a variety of situations and tasks (Logan, 1994; van Boxtel et al., 2001). Given this correlation, it is noteworthy that the go processes (tracking PE and go-RT) were dif- ferent, with the correlation between them being low and nonsignificant. Moreover, the correlations between each go measure and all other measures were dissimilar. ${ }^{5}$ The differences between the go measures are important since they demonstrate that the tasks were distinct. This, in turn, emphasizes the finding that the stop measures were highly correlated despite the task differences.

Despite the high correlation between the SSRTs, it could be argued that the .84 correlation measured a general component not specific to response inhibition - that is, one that is also present in go-RT. Indeed, although not significant, go-RT did correlate positively with the stopping measures. To test whether tracking SSRT included a component specific to response inhibition, a hierarchal regression analysis was performed with go-RT introduced first, followed by tracking SSRT. Go-RT accounted for $37 \%$ of the variance in countermanding SSRT, with tracking SSRT accounting for an additional 35\% $(p<.05)$. In contrast, when tracking SSRT was introduced first to the regression, $71 \%$ of the variance of SSRT was accounted

Table 1

Correlations Between the Dependent Measures of Both the Countermanding Task and the Continuous Tracking Task

\begin{tabular}{lrrrrrrr}
\hline & 2 & 3 & 4 & 5 & 6 & $M$ & $S D$ \\
\hline 1. Countermanding go-RT & .53 & .61 & .61 & .61 & -.12 & 432.8 & 38.3 \\
2. Countermanding SSRT $_{1}$ & & $.72 *$ & $.93^{*}$ & $.81^{*}$ & .49 & 237.6 & 24.1 \\
3. Countermanding SSRT $_{2}$ & & & $.92^{*}$ & $.75^{*}$ & .34 & 223.5 & 22.8 \\
4. Countermanding mean SSRT & & & & $.84^{*}$ & .45 & 230.5 & 21.7 \\
5. Continuous tracking SSRT & & & & & -.31 & 261.4 & 27.5 \\
6. Continuous tracking phase error & & & & & & 7.24 & 3.07 \\
\hline
\end{tabular}

Note-All measures are in milliseconds, other than continuous tracking phase error, which is in degrees. SSRT, stop signal reaction time. $* p<.05$. 
for. This demonstrates that the two SSRT measures are sensitive to a mechanism that appears to be specific to response inhibition.

\section{Are There Meaningful Differences Between the Tasks?}

One might perhaps argue that the high correlation between countermanding and tracking SSRTs occurred merely because the tasks did not differ in a meaningful way. For instance, one might suggest that the manipulation of task failed to engage different forms of stopping, since once a tracking response is initiated it is "locked in" and no longer constitutes an ongoing response. Two analyses addressed, and rejected, this idea. First, if one response is merely prepared and the other actually ongoing, it follows that SSRT should be significantly longer for the latter, because of the additional time required to physically implement the inhibition of an ongoing response (e.g., Mills, 1999). A $t$ test comparing countermanding SSRT and tracking SSRT was significant $[t(9)=$ $6.5, p<.001]$, reflecting the fact that tracking SSRT was longer than countermanding SSRT by approximately $30 \mathrm{msec}$ (Table 1).

The second analysis examined whether the delay between the go signal and the stop signal had similar effects on countermanding and tracking inhibition. It is well established that in the countermanding task, SSRT may decline significantly as the delay between the go and the stop signals lengthens. This decline is generally understood to reflect the race between go and stop responses. As the delay increases, only shorter stop latencies win the race, and longer ones contribute less to SSRT (Band et al., 2003; Logan \& Burkell, 1986). For a tracking response, however, there is no race between go and stop processes, because the go response is already occurring. As a result, SSRT should not decline as the delay between go and stop signals increases.

This is the pattern of results that was found (Table 2). An analysis of variance (ANOVA) revealed that delay had a significant effect on $\mathrm{SSRT}_{1}\left[F(3,27)=68.4, M S_{\mathrm{e}}=\right.$ $458, p<.01]$. As the delay increased, $\mathrm{SSRT}_{1}$ decreased, and all Newman-Keuls comparisons were significant $(p<.05)$. In contrast, longer stop signal delays did not lead to shorter tracking SSRTs. In fact, an ANOVA indicated that tracking SSRT was shortest at the 30 -msec delay $\left[F(3,27)=4.7, M S_{\mathrm{e}}=384, p<.01\right]$. This was confirmed by Newman-Keuls comparisons (no other comparison was significant). It is likely that the onset of the flash (which lasted for $100 \mathrm{msec}$ and was, therefore,

Table 2

Mean Stop Signal Reaction Times (in Milliseconds) as a Function of Delay in the Countermanding and Tracking Tasks

\begin{tabular}{lcccc}
\hline \multirow{2}{*}{\multicolumn{1}{c}{ Task }} & 30 & 110 & 190 & 270 \\
\cline { 2 - 5 } & 308 & 253 & 212 & 178 \\
Countermanding & 243 & 266 & 262 & 274 \\
\hline
\end{tabular}

offset in the other delays) led to increased preparedness to stop and to alerting (Slater-Hammel, 1960).

Interestingly, the tracking task also sheds light on the influence of delay on countermanding SSRT (e.g., Logan \& Burkell, 1986; Logan \& Cowan, 1984). Alternative accounts of the race horse account described above attribute the decline in SSRT with increases in delay to a refractory effect at short SOAs or to an increased preparedness to stop at longer delays (Band et al., 2003; Logan \& Burkell, 1986). These alternatives predict that tracking SSRT should also decrease as delay increases, which it did not do (although one might argue that a refractory effect was absent because the flash was not sufficiently response relevant in the tracking task). In sum, it seems reasonable to conclude that although the two SSRT measures appear to be sensitive to the same inhibition process, they are susceptible to different processes afforded by the particular context and demands of the task.

In addition to addressing different hypotheses set forth from the countermanding procedure the tracking task has several useful advantages. For example, it does not require the assumption that stop and go processes are independent, and SSRT data are collected directly on every trial (Morein-Zamir \& Meiran, 2003). The latter advantage may prove particularly useful when testing under time constraints, since fewer trials are needed. Furthermore, measures of tracking SSRT variability are easily accessible. Recently, Band et al. (2003) indicated that the countermanding procedure cannot provide a reasonable estimate of response inhibition variability, which is believed to be indicative of deficient response inhibition, such as that found in ADHD (Oosterlaan et al., 1998).

\section{Response Inhibition in the Tracking Task}

It could be argued that the release of force measured by tracking SSRT should be interpreted as an action, rather than as the termination of an ongoing action. This is especially true for the present version of the tracking task, modified from Morein-Zamir and Meiran (2003). In accord with this argument, Naito and Matsumura (1996) found no significant difference when countermanding constituted refraining from a buttonpress versus refraining from the release of a pressed key. In the present study, detracting pressure had important visual consequences: The speed of the response stimulus decreased, and when pressure returned to baseline (resting force), the stimulus stopped. There are several reasons why stopping here can be considered response inhibition. First, the coding of the response is directly and continuously contingent on the consequences of the actions of the participant. In particular, releasing the pressure causes the response marker to stop moving. Furthermore, by coupling resident effects (the proprioceptive and tactile cues from the finger) with the remote effects (the movement of a visual stimulus), the participants would perceive increased pressure as an action and the release of pressure as a termination of this action (Hommel et al., 2001). For the same reason, the participants never lifted their fingers from the key, ensuring that they did not per- 
ceive another action (stop change) as stopping the marker. Ladefoged et al. (1973) made similar assumptions when studying the termination of speech in response to an external signal (see also Logan, 1982, who examined the inhibition of typing). Ultimately, the results support our interpretation that the participants coded the release of pressure as an action termination.

\section{Conclusion}

The present study demonstrated that the countermanding and continuous-tracking tasks measure a similar stopping process, supporting the hypotheses that response withholding and response termination comprise the same general response inhibition mechanism. This allows one to generalize the findings of one task to the other and lends converging validity to both. Thus, this study supports the notion that there exists a general mechanism for motor response inhibition. It remains to be seen whether this response inhibition is part of a unitary inhibitory process that generalizes to different effectors and action complexities. One may also question how this inhibitory process relates to other executive functions. On the one hand, behavioral inhibition has been hypothesized to be distinct from cognitive inhibition (including negative priming and directed forgetting) and interference control (including Stroop, flanker, and dual tasks), although all are considered executive, or effortful, inhibition (Nigg, 2000). Alternatively, there is evidence stressing a general executive function called inhibition that encompasses motor inhibition, as well as inhibition found in the Stroop and antisaccade tasks (Miyake et al., 2000). Hence, the relationship between the present examples of response inhibition and seemingly related tasks remains to be uncovered.

\section{REFERENCES}

BAND, G. P. H., \& van BoxTEL, G. J. M. (1999). Inhibitory motor control in stop paradigms: Review and reinterpretation of neural mechanisms. Acta Psychologica, 101, 179-211.

Band, G. P. H., VAN DER Molen, M. W., \& Logan, G. D. (2003). Horserace model simulations of the stop-signal procedure. Acta Psychologica, 112, 105-142.

BARKLEY, R. A. (1997). Behavioral inhibition, sustained attention, and executive functions: Constructing a unifying theory of ADHD. Psychological Bulletin, 121, 65-94.

DE Jong, R., Coles, M. G. H., \& Logan, G. D. (1995). Strategies and mechanisms in nonselective and selective inhibitory motor control. Journal of Experimental Psychology: Human Perception \& Performance, 21, 498-511.

Henry, F. M., \& Harrison, J. S. (1961). Refractoriness of a fast movement. Perceptual \& Motor Skills, 13, 351-354.

Hommel, B., Müsseler, J., Aschersleben, G., \& Prinz, W. (2001). The theory of event coding (TEC): A framework for perception and action planning. Behavioral \& Brain Sciences, 24, 849-878.

KoK, A. (1999). Varieties of inhibition: Manifestations in cognition, event-related potentials and aging. Acta Psychologica, 101, 129-158.

Kramer, A. F., Humphrey, D. G., Larish, J. F., Logan, G. D., \& STRAYER, D. L. (1994). Aging and inhibition: Beyond a unitary view of inhibitory processing in attention. Psychology \& Aging, 9, 491512 .

Ladefoged, P., Silverstein, R., \& Papcun, G. (1973). Letter: Interruptibility of speech. Journal of the Acoustical Society of America, 54, 1105-1108.
LAPPIN, J. S., \& ERIKSEN, C. W. (1966). Use of a delayed signal to stop a visual reaction-time response. Journal of Experimental Psychology, 72, 805-811.

LoGAN, G. D. (1982). On the ability to inhibit complex movements: A stop-signal study of typewriting. Journal of Experimental Psychology: Human Perception \& Performance, 8, 778-792.

Logan, G. D. (1994). On the ability to inhibit thought and action: A users' guide to the stop signal paradigm. In D. Dagenbach \& T. H. Carr (Eds.), Inhibitory processes in attention, memory, and language (pp. 214-249). San Diego: Academic Press.

Logan, G. D., \& Burkell, J. (1986). Dependence and independence in responding to double stimulation: A comparison of stop, change and dual-task paradigms. Journal of Experimental Psychology: Human Perception \& Performance, 12, 549-563.

LogAn, G. D., \& Cowan, W. B. (1984). On the ability to inhibit thought and action: A theory of an act of control. Psychological Review, $\mathbf{9 1}_{2}$ 295-327.

Logan, G. D., Cowan, W. B., \& Davis, K. A. (1984). On the ability to inhibit simple and choice reaction time responses: A model and a method. Journal of Experimental Psychology: Human Perception \& Performance, 10, 276-291.

McGarry, T., InGLIS, J. T., \& Franks, I. M. (2000). Against a final ballistic process in the control of voluntary action: Evidence using the Hoffmann reflex. Motor Control, 4, 469-485.

Mills, K. R. (1999). Magnetic stimulation of the human nervous system. New York: Oxford University Press.

Miyake, A., Friedman, N. P., Emerson, M. J., Witzki, A. H., HowERTER, A., \& WAGER, T. D. (2000). The unity and diversity of executive functions and their contributions to complex "frontal lobe" tasks: A latent variable analysis. Cognitive Psychology, 41, 49-100.

Morein-Zamir, S., \& MeIran, N. (2003). Individual stopping times and cognitive control: Converging evidence from a new stop signal paradigm. Quarterly Journal of Experimental Psychology, 56, 469490.

Naito, E., \& Matsumura, M. (1996). Movement-related potentials associated with motor inhibition under different preparatory states during performance of two visual stop signal paradigms in humans. Neuropsychologia, 34, 565-573.

NigG, J. T. (2000). On inhibition/disinhibition in developmental psychopathology: Views from cognitive and personality psychology and a working inhibition taxonomy. Psychological Bulletin, 126, 220246.

Oosterlaan, J., Logan, G. D., \& Sergeant, J. A. (1998). Response inhibition in $\mathrm{AD} / \mathrm{HD}, \mathrm{CD}$, comorbid $\mathrm{AD} / \mathrm{HD}+\mathrm{CD}$, anxious, and control children: A meta-analysis of studies with the stop task. Journal of Child Psychology \& Psychiatry, 39, 411-425.

Osman, A., Kornblum, S., \& Meyer, D. E. (1990). Does motor programming necessitate response execution? Journal of Experimental Psychology: Human Perception \& Performance, 16, 183-198.

SLATER-HAMmEL, A. T. (1960). Reliability, accuracy and refractoriness of a transit reaction. Research Quarterly, 31, 217-228.

van Boxtel, G. J. M., van der Molen, M. W., Jennings, J. R., \& Brunia, C. H. M. (2001). A psychophysiological analysis of inhibitory motor control in the stop-signal paradigm. Biological Psychology, 58, 229-262.

\section{NOTES}

1. De Jong et al. (1995) suggested a more complex inhibition mechanism involving central and peripheral stopping. However, their results can also be explained by a single stop mechanism (Band \& van Boxtel, 1999; van Boxtel et al., 2001).

2. To compute SSRTs using the race model, one must assume independence between stop and go processes. Since the present go task is different from the more common choice tasks, we tested for independence between go-RTs and failures to respond (signal-respond) RTs (Logan, 1994; although see Band et al., 2003, for a critique of independence tests). The results revealed that stop-respond RTs were significantly shorter than go-RTs, and that stop-respond RTs increased at longer delays in accordance with independence predictions.

Furthermore, there were no significant differences between the observed stop-respond and stop-respond values predicted from go-RTs at 
the longest 3 delays (the low number of observations in the shortest delay precluded it from analysis).

3. In agreement with Morein-Zamir and Meiran (2003), final RT (the time to complete a stop) was not a reliable measure of response inhibition in the tracking task, correlating significantly only with tracking PE $(r=.83, p<.05)$.

4. Interestingly, tracking SSRT had higher correlations with both countermanding SSRT measures $(r=.81$ and $r=.75)$ than countermanding SSRTs had between themselves $(r=.72)$, indicating that tracking SSRT is highly reliable.
5. We tentatively propose that the correlation between countermanding SSRT and tracking PE reflects a shared general source of executive control. Tracking necessitates a continuous goal-directed response that is continuously monitored and adjusted (Barkley, 1997). Nevertheless, tracking SSRT accounted for another $32 \%$ of countermanding variance over and above this component.

(Manuscript received August 6, 2003;

revision accepted for publication January 28, 2004.) 